\title{
Exact Simulation and Sensitivity Analysis of Multiplexing Networks
}

\author{
JOHN W. BANDLER, FELLOW, IEEE, SHAHROKH DAIJAVAD, STUDENT MEMBER, IEEE, \\ AND QI-JUN ZHANG, STUDENT MEMBER, IEEE
}

\begin{abstract}
This paper presents a novel approach to the simulation and sensitivity analysis of multiplexing networks. All computations are performed efficiently utilizing the concept of forward and reverse analysis which is elegant and effective in cascaded circuit analysis. Formulas are derived for such responses as input or output reflection coefficient, common port and channel output port return losses, insertion loss, gain slope, and group delay. Exact sensitivities w.r.t. all variables of interest, including frequency, are evaluated. The fundamental assumption is that the transmission matrices for the individual components of the network and their sensitivities w.r.t. possible variables inside them are available. An explicit algorithm is provided describing the details of the computational aspects of our theory. The formulas are applied to the optimal design of practical contiguous or noncontiguous band multiplexers consisting of multicavity filters distributed along a waveguide manifold. An example of optimizing a practical 12 channel, 12-GHz contiguous band multiplexer without dummy channels, which is the state-of-the-art structure used as the output multiplexer in satellite transponders, is presented.
\end{abstract}

\section{INTRODUCTION}

$\mathrm{T}$ HE DESIGN OF contiguous band multiplexers consisting of multicoupled cavity filters was a problem of significant theoretical interest for several years [1], [2]; however, the manufacturing of such structures with more than five channels did not appear to be feasible. Recently, the subject has turned into an important development area in microwave engineering practice due to reports by leading manufacturers of successful production of 12-channel contiguous band multiplexers for satellite applications [3]-[6]. The employment of optimization techniques to determine the best multiplexer parameters has been an indispensable part of the design procedures reported [4]-[9]. The use of a powerful gradient-based minimax optimization technique [8], [9] has reduced significantly the CPU time required in the design procedure.

The implementation of a gradient-based optimization technique in multiplexer design requires, as a vital step, a robust and efficient algorithm for simulation and sensitivity analysis. In this paper, we present a new and elegant approach to the simulation of such responses as common port and channel output port return losses, insertion loss,

Manuscript received March 27, 1985; revised July 26, 1985. This work was supported in part by the Natural Sciences and Engineering Research Council of Canada under Grant G1135.

The authors are with the Simulation Optimization Systems Research Laboratory and the Department of Electrical and Computer Engineering, McMaster Unıversity, Hamilton, Canada, L8S 4L7.

J. W. Bandler is also with Optimization Systems Associates, 163 Watson's Lane, Dundas, Ontario, Canada L9H 6L1.

IEEE Log Number 8405939. and group delay between source and channel output ports and their first-order sensitivities w.r.t. all network parameters and frequency. Our approach can be applied to any network in the general category of multiplexing or branched cascaded structures [10]. Each basic component of the structure is either a 2-port model or a 3-port junction and can contain variables or be constant. The fundamental requirement for the approach is that the transmission matrix description of all basic components and their derivatives, if they contain variables, are provided. This information is utilized in a systematic and efficient scheme which leads to the evaluation of various responses of the network at all ports of interest. An important feature of our approach is that the network structure is fully exploited and an effective cascaded analysis method, namely, the forward and reverse analysis method [11], [12], is extended and applied. Components of various complexity are permitted and nonideal effects such as losses and dispersion can be directly taken into account. The approach handles contiguous and noncontiguous band designs in a unified manner.

The presentation is organized in the following way. In Section II, we describe the basic cascaded analysis approach as applied to a general branched cascaded circuit. Formulas for Thevenin equivalents, reflection coefficients and branch output voltages as well as their first- and second-order sensitivities w.r.t. design variables and frequency at any reference plane are developed. In Section III, we consider multiplexers consisting of multicavity filters distributed along a waveguide manifold. Transmission matrices and sensitivity expressions for typical components in a multiplexer, which are required by our approach, are tabulated. Response and sensitivity formulas, applicable in the multiplexer design, are also listed. In Section IV, an algorithm based on the theory discussed in Sections II and III is presented. Finally, in Section V, we consider a practical 12-channel $12-\mathrm{GHz}$ multiplexer and apply our theory to optimize such a structure, taking into account many nonideal effects, using a gradient-based optimization procedure.

\section{BRanched CASCADED Networks}

A multiplexer falls into the category of a general class of networks, namely, branched cascaded structures as shown in Fig. 1. For such structures, we develop a novel procedure to calculate the reflection coefficients at the common 


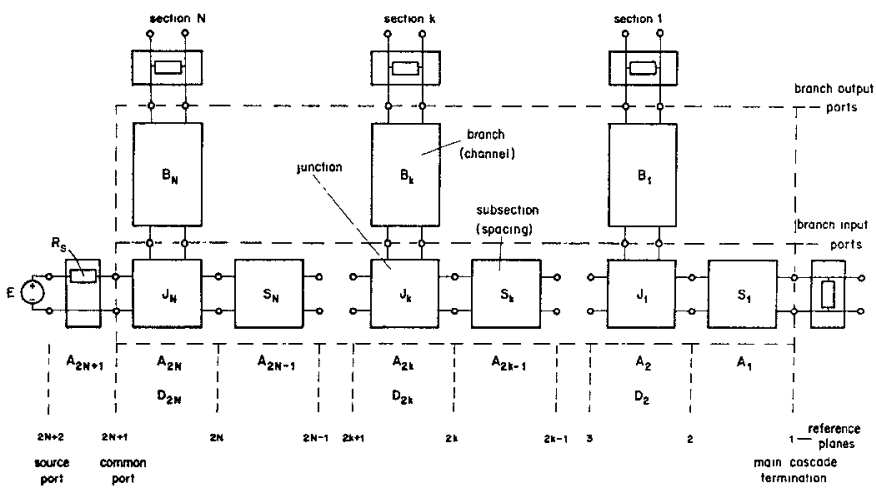

Fig. 1. The branched cascaded network under consideration. $J_{1}, J_{2}, \cdots, J_{N}$ are arbitrarily defined 3-port junctions. $B_{1}, B_{2}, \cdots, B_{N}$ are branches or channels which may each be represented in reduced cascade form and $S_{1}, S_{2}, \cdots, S_{N}$ represent the subsections between junctions. Principal concepts of reference planes, transmission matrices, and typical ports are illustrated.

port and branch output ports as well as branch output voltages. Simultaneously, first- and second-order derivatives are evaluated. The approach is based on the computation of Thevenin source and impedance equivalents and their first- and second-order sensitivities w.r.t. design parameters and frequency at the ports of interest.

\section{A. Models of Basic Components}

Although the basic components of a branched cascaded circuit are 2-port elements or 3-port junctions, internally they can be complicated subnetworks characterized by admittance, impedance, or hybrid matrices. An example of such a subnetwork is the multicoupled cavity filter described by an impedance matrix and containing many design variables. As a prerequisite step towards using our theory, the transmission matrix for each 2-port element should be deduced either by a reduction procedure or by direct measurements. Also, if variables exist in a subnetwork, the derivative of the corresponding transmission matrix should be provided. For the 3-port junctions, however, a 3-port description in the form of an arbitrary hybrid matrix, is sufficient.

\section{B. Reference Planes}

Consider the branched cascaded network of Fig. 1, which consists of $N$ sections. A typical section, e.g., the $k$ th one, has a junction, $n(k)$ cascaded elements of branch $k$, and a subsection along the main cascade, as shown in Fig. 2. All reference planes in the entire network are defined uniformly and numbered consecutively beginning from the main cascade termination, which is designated reference plane 1 . The source port is at reference plane $2 N+2$. The termination of the $k$ th branch is called reference plane $\tau(k)$ and the branch main cascade connection (branch input port) is reference plane $\sigma(k), k=1,2, \cdots, N$, where

$$
\begin{aligned}
\tau(1) & =2 N+3 & & \\
\sigma(k) & =\tau(k)+n(k), & & k=1,2, \cdots, N \\
\tau(k) & =\sigma(k-1)+1, & & k=2,3, \cdots, N .
\end{aligned}
$$

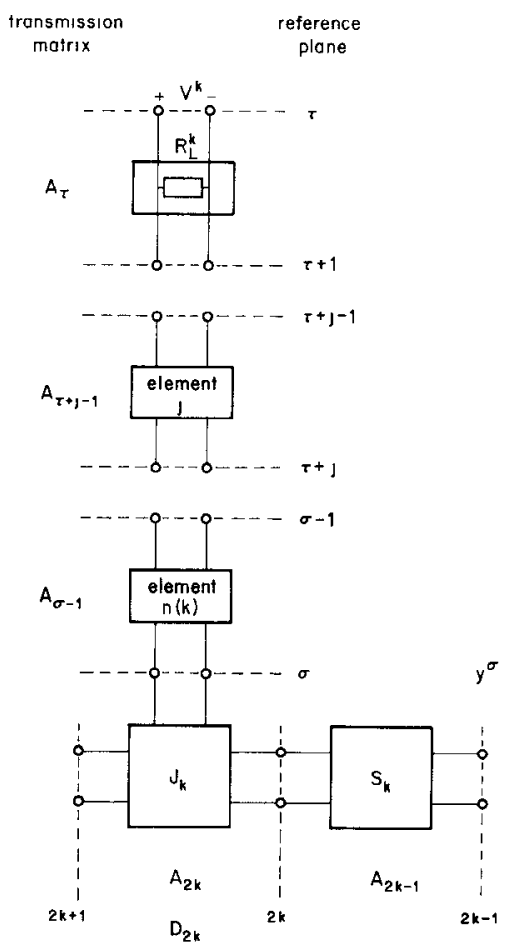

Fig. 2. Detail of the $k$ th section of a branched cascaded circuit showing reference planes along the branch where $\tau=\tau(k)$ and $\sigma=\sigma(k)$.

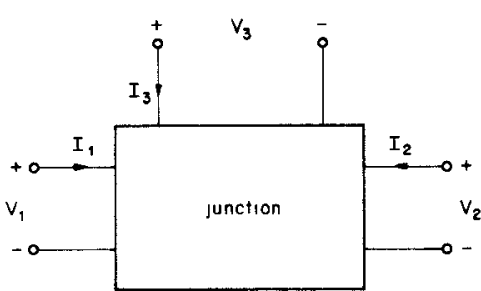

Fig 3. A 3-port junction in which ports 1 and 2 are considered along a main cascade and port 3 represents a channel or branch of the main cascade

\section{Reduction of Junctions to 2-Port Representations}

Bandler et al. [11], [12] introduced the concept of forward and reverse analysis for cascaded networks. To simplify the structure under consideration to a cascade of 2-ports for which the forward and reverse analysis is applicable, the 3-port junctions are reduced to 2-port representations.

Consider the 3-port junction shown in Fig. 3. To carry the analysis through the junction along the main cascade, we terminate port 3 , e.g., by calculating the equivalent admittance seen at this port given by $Y_{3}=\left(-I_{3}\right) / V_{3}$ and represent the transmission matrix between ports 1 and 2 by $\boldsymbol{A}$. The analysis can also be carried through the junction into the branch by terminating port 2 , e.g., calculating $Y_{2}=\left(-I_{2}\right) / V_{2}$ and denoting the transmission matrix between ports 1 and 3 by $D$.

As an example, suppose the 3-port junction is characterized by a hybrid matrix $\boldsymbol{H}$ such that

$$
\left[\begin{array}{lll}
V_{1} & I_{1} & I_{3}
\end{array}\right]^{T}=\boldsymbol{H}\left[\begin{array}{lll}
V_{2} & I_{2} & V_{3}
\end{array}\right]^{T}
$$


where $\boldsymbol{H}=\left[h_{i j}\right]_{3 \times 3}$. Then $\boldsymbol{A}=\left[a_{i j}\right]_{2 \times 2}$ can be found from

$$
a_{i j}=(-1)^{j-1}\left[h_{i j}-h_{i 3} h_{3 j} /\left(Y_{3}+h_{33}\right)\right] .
$$

For various forms of hybrid matrices $\boldsymbol{H}$, the 2-port representation $\boldsymbol{A}$ or $\boldsymbol{D}$ is evaluated in a similar manner using elements of $\boldsymbol{H}$ and the equivalent termination at port 3 or 2 .

\section{Cascaded Analysis}

Having reduced the junctions to 2-port representations, the network structure between any two reference planes is transformed to a simple cascade of two-ports. Assuming that the transmission matrices for all 2-ports are given, we define the equivalent transmission matrix between reference planes $i$ and $j$ by

$$
\boldsymbol{Q}_{i j} \triangleq\left[\begin{array}{ll}
\boldsymbol{p}_{i j} & \boldsymbol{q}_{\imath j}
\end{array}\right] \triangleq\left[\begin{array}{ll}
A_{\imath \jmath} & B_{i j} \\
C_{\imath j} & D_{i j}
\end{array}\right]
$$

where

$$
\boldsymbol{p}_{i j} \triangleq\left[\begin{array}{l}
A_{i j} \\
C_{i j}
\end{array}\right], \quad \boldsymbol{q}_{i j} \triangleq\left[\begin{array}{c}
B_{i j} \\
D_{i j}
\end{array}\right] .
$$

In a forward (reverse) analysis, $Q_{i j}$ is computed by initializing row vectors $\boldsymbol{e}_{1}^{T}$ and $\boldsymbol{e}_{2}^{T}$ (column vectors $\boldsymbol{e}_{1}$ and $\boldsymbol{e}_{2}$ ) at reference plane $i(j)$ and successively premultiplying (postmultiplying) each transmission matrix by the resulting row (column) vector until reference plane $j(i)$ is reached. $e_{1}$ and $e_{2}$ are unit vectors given by $\left[\begin{array}{ll}1 & 0\end{array}\right]^{T}$ and $\left[\begin{array}{ll}0 & 1\end{array}\right]^{T}$, respectively.

Let $\phi$ be a generic notation that can be used to represent any design variable in the network. Sensitivities of $\boldsymbol{Q}_{t j}$ w.r.t. any variable $\phi$ located between reference planes $i$ and $j$ are evaluated as

$$
\frac{\partial \boldsymbol{Q}_{l j}}{\partial \phi}=\sum_{l \in I_{\phi}} \frac{\partial}{\partial \phi}\left(\boldsymbol{Q}_{i j}^{l}\right)
$$

where $I_{\phi}$ is an index set whose elements identify the transmission matrices containing $\phi$ and $\partial Q_{t_{j}}^{l} / \partial \phi$ is the result of a forward or reverse analysis between reference planes $i$ and $j$ with the $l$ th matrix replaced by its derivative w.r.t. $\phi$. Second-order sensitivities can be derived in a similar manner as

$$
\frac{\partial^{2} \boldsymbol{Q}_{i j}}{\partial \phi \partial \omega}=\sum_{l \in I_{\phi}} \sum_{m \in I_{\omega}} \frac{\partial^{2} \boldsymbol{Q}_{l j}^{l m}}{\partial \phi \partial \omega}
$$

where $I_{\phi}$ and $I_{\omega}$ are index sets, not necessarily disjoint, identifying those matrices which are functions of $\phi$ and $\omega$. Also, we define $\partial^{2} \boldsymbol{Q}_{i j}^{\prime m} /(\partial \phi \partial \omega)$ as the second-order sensitivity of $\boldsymbol{Q}_{\imath \jmath}$ as if $\phi$ and $\omega$ exist only in the $l$ th and $m$ th matrices, respectively.

\section{E. Thevenin Equivalent Circuits and Basic Responses}

To calculate the input reflection coefficient at the common port, the output reflection coefficients at the branch output ports, as well as the branch output voltages and their sensitivities in a unified manner, we employ Thevenin

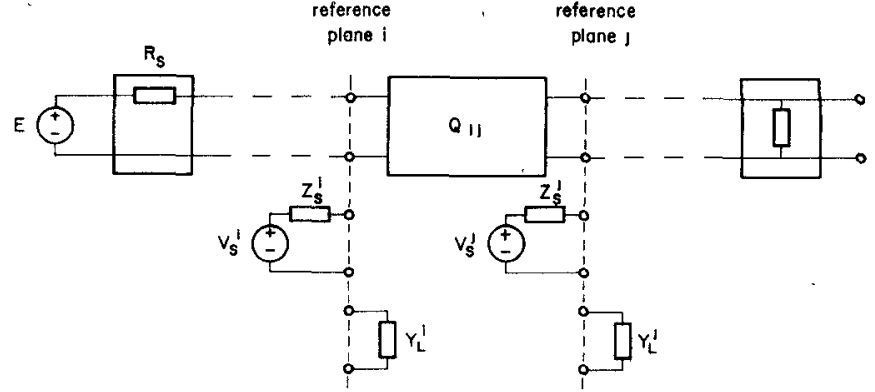

(a)

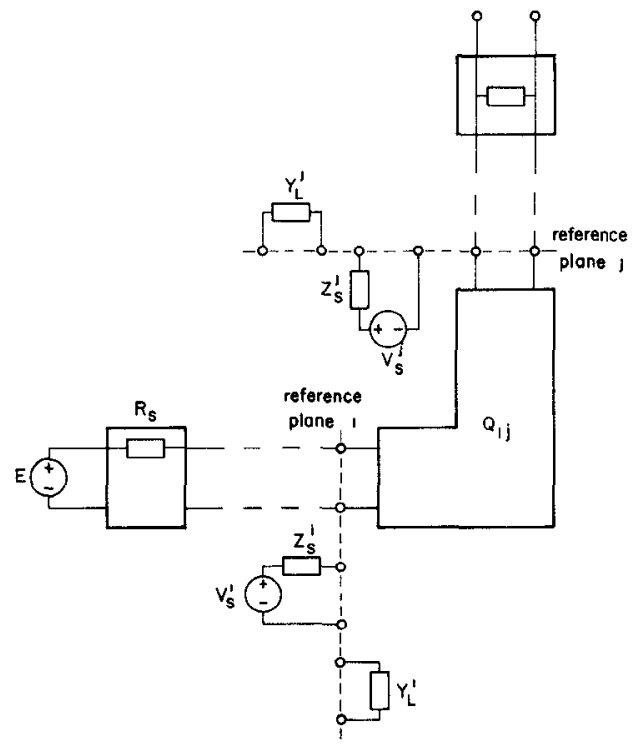

(b)

Fig. 4. Thevenin and Norton equivalents at reference planes $i$ and $j$, where reference plane $i$ is towards the source w.r.t. reference plane $j$. (a) Reference plane $j$ is in the main cascade. (b) Reference plane $j$ is in a branch.

equivalents at the ports of interest evaluated by the method of forward and reverse analysis [11]. Denoting the Thevenin equivalent voltages and impedances at reference planes $i$ and $j$ by $V_{S}^{l}, Z_{S}^{l}, V_{S}^{\prime}$, and $Z_{S}^{\prime}$, we have

$$
V_{S}^{j}=\frac{V_{S}^{l}}{A_{\imath j}+Z_{S}^{i} C_{\imath j}}
$$

and

$$
Z_{S}^{j}=\frac{B_{\imath j}+Z_{S}^{i} D_{i j}}{A_{\imath j}+Z_{S}^{i} C_{\imath j}}
$$

where reference plane $i$ is located towards the source w.r.t. $j$, as shown in Fig. 4. The sensitivities are obtained as

$$
\left(V_{S}^{J}\right)_{\phi}=\frac{\left(V_{S}^{t}\right)_{\phi}-\left[\left(A_{i j}\right)_{\phi}+Z_{S}^{i}\left(C_{i j}\right)_{\phi}+\left(Z_{S}^{i}\right)_{\phi} C_{\imath j}\right] V_{S}^{J}}{A_{\imath j}+Z_{S}^{i} C_{\imath j}}
$$

and

$$
\left(Z_{S}^{j}\right)_{\phi}=\frac{\left[\begin{array}{ll}
1 & Z_{S}^{i}
\end{array}\right]\left(\boldsymbol{Q}_{\imath \jmath}\right)_{\phi}\left[\begin{array}{c}
-Z_{S}^{\jmath} \\
1
\end{array}\right]+\left(Z_{S}^{i}\right)_{\phi}\left(D_{\iota_{J}}-Z_{S}^{J} C_{i_{j}}\right)}{A_{i j}+Z_{S}^{i} C_{\imath \jmath}}
$$


where subscript $\phi$ denotes $\partial / \partial \phi$.

If the reflection coefficient at the $k$ th branch output port and its sensitivities are to be calculated, then (9) and (11) are specialized to

$$
Z_{S}^{\tau+1}=\frac{B}{A}
$$

and

$$
\left(Z_{S}^{\tau+1}\right)_{\phi}=\frac{(B)_{\phi}-(A)_{\phi} Z_{S}^{\tau+1}}{A}
$$

where $A \equiv A_{2 N+2, \tau+1}, B \equiv B_{2 N+2, \tau+1}$, and $\tau \equiv \tau(k)$. This is simply due to the fact that there is no impedance to the left of reference plane $2 N+2$, i.e., $Z_{S}^{2 N+2}=0$. The corresponding output reflection coefficient is defined as

$$
\rho^{k} \triangleq \frac{Z_{S}^{\tau+1}-R_{L}^{k}}{Z_{S}^{\tau+1}+R_{L}^{k}}
$$

where $R_{I}^{k}$ is the load resistance at the $k$ th channel output. Clearly, (13) is utilized in the evaluation of $\left(\rho^{k}\right)_{\phi}$ as

$$
\left(\rho^{k}\right)_{\phi}=\frac{\left(Z_{S}^{\tau+1}\right)_{\phi}\left(1-\rho^{k}\right)}{Z_{S}^{\tau+1}+R_{L}^{k}} .
$$

Channel output voltage is also computed by utilizing the Thevenin equivalent voltage source and impedance at the branch output port. At the $k$ th channel, we have

$$
V^{k}=\frac{R_{L}^{k}}{A\left(R_{L}^{k}+Z_{S}^{\tau+1}\right)}
$$

assuming a normalized excitation at the source port. This can be explained easily by noticing that $V^{k}$ is evaluated using a voltage divider once $V_{S}^{\tau+1}$ is known. Using (8) and taking into account that $V_{S}^{2 N+2}=1$ and $Z_{S}^{2 N+2}=0$, we have $V_{S}^{\tau+1}=1 / A$. Also

$$
\left(V^{k}\right)_{\phi}=-R_{L}^{k} V^{k}\left[\frac{(A)_{\phi}}{A}+\frac{\left(Z_{S}^{\tau+1}\right)_{\phi}}{R_{L}^{k}+Z_{S}^{\tau+1}}\right] .
$$

The second-order sensitivity of $V^{k}$ w.r.t. $\phi$ and $\omega$, i.e., $\partial^{2} V^{k} /(\partial \phi \partial \omega)$, is obtained via evaluation of $\partial^{2} Z_{S}^{++1} /(\partial \phi \partial \omega)$. Substituting $\omega$ for $\phi$ in (13) and differentiating w.r.t. $\phi$, gives

$$
\begin{aligned}
& \left(Z_{S}^{\tau+1}\right)_{\phi \omega} \\
& =\frac{(B)_{\phi \omega}-Z_{S}^{\tau+1}(A)_{\phi \omega}-(A)_{\omega}\left(Z_{S}^{\tau+1}\right)_{\phi}-\left(Z_{S}^{\tau+1}\right)_{\omega}(A)_{\phi}}{A}
\end{aligned}
$$

where double subscript $\phi \omega$ denotes $\partial^{2} /(\partial \phi \partial \omega)$.

Now, replacing $\phi$ by $\omega$ in (17) and differentiating w.r.t. $\phi$, we have

$$
\begin{array}{r}
\left(V^{k}\right)_{\phi \omega}=\frac{\left(V^{k}\right)_{\phi}\left(V^{k}\right)_{\omega}}{V^{k}}-R_{L}^{k} V^{k}\left[\frac{A(A)_{\phi \omega}-A_{\phi} A_{\omega}}{A^{2}}\right. \\
\left.+\frac{\left(Z_{S}^{\tau+1}\right)_{\phi \omega}\left(R_{L}^{k}+Z_{S}^{\tau+1}\right)-\left(Z_{S}^{\tau+1}\right)_{\omega}\left(Z_{S}^{\tau+1}\right)_{\phi}}{\left(R_{L}^{k}+Z_{S}^{\tau+1}\right)^{2}}\right] .
\end{array}
$$

Norton equivalent admittances and current sources are calculated similarly to the Thevenin equivalents. Denoting the Norton equivalent currents and admittances at reference planes $i$ and $j$ by $I_{L}^{l}, Y_{L}^{l}, I_{L}^{\prime}$, and $Y_{L}^{j}$, we have

$$
Y_{L}^{i}=\frac{C_{t \jmath}+Y_{L}^{\jmath} D_{\imath \jmath}}{A_{t \jmath}+Y_{L}^{\prime} B_{l \jmath}}
$$

and

$$
I_{L}^{l}=I_{L}^{J}=0
$$

Also

$$
\left(Y_{L}^{\imath}\right)_{\phi}=\frac{\left[\begin{array}{ll}
-Y_{L}^{\imath} & 1
\end{array}\right]\left(\boldsymbol{Q}_{\imath \jmath}\right)_{\phi}\left[\begin{array}{c}
1 \\
Y_{L}
\end{array}\right]+\left(Y_{L}^{J}\right)_{\phi}\left(D_{\imath \jmath}-Y_{L}^{\imath} B_{\imath \jmath}\right)}{A_{\imath \jmath}+Y_{L}^{\prime} B_{\imath \jmath}} .
$$

As special cases of (20), the equivalent admittances $Y_{3}$ and $Y_{2}$ required in the reduction of junctions to 2-port representations are calculated as

$$
Y_{3}^{k}=Y_{L}^{\sigma(k)}=\frac{C_{\sigma(k), \tau(k)}}{A_{\sigma(k), \tau(k)}}, \quad k=1,2, \cdots, N
$$

and, for a short-circuit main cascade termination

$$
Y_{2}^{k}=Y_{L}^{2 k}=\frac{D_{2 k, 1}}{B_{2 k, 1}}, \quad k=1,2, \cdots, N
$$

The common-port reflection coefficient is also computed using the Norton equivalent (at the source reference plane) as

$$
\rho^{0}=1-\frac{2 R_{S} D_{2 N+2,1}}{B_{2 N+2,1}} .
$$

Its sensitivity is given by

$$
\left(\rho^{0}\right)_{\phi}=2 R_{S} \frac{(B)_{\phi} D-(D)_{\phi} B}{B^{2}}
$$

where $B \equiv B_{2 N+2,1}$ and $D \equiv D_{2 N+2,1}$.

\section{ANAlysis of Specific Multiplexer STructures}

While the approach developed in Section II is general, as a special case, the design of multiplexers consisting of coupled cavity filters distributed along a waveguide manifold is considered here in more detail. Contiguous or noncontiguous band multiplexers are treated in a similar manner. Fig. 5, which is a special case for the structure in Fig. 1, illustrates a typical circuit equivalent for a multiplexer. A branch consists of a coupled-cavity filter, together with input-output transformers, and an impedance inverter. A subsection is the waveguide section separating two adjacent filters, and the junction is the equivalent circuit model for the physical junction between channel filters and the manifold. The main cascade is short circuited and the responses of interest are common-port return loss, channel output return loss, insertion or trans- 


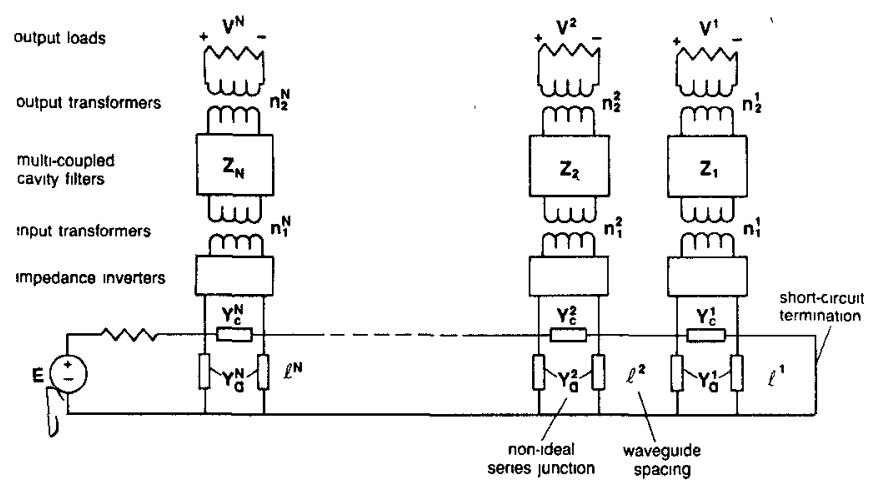

Fig. 5. Equivalent circuit of a contiguous band multiplexer. Each channel has a multicoupled cavity filter with input and output transformers as well as an impedance inverter. The main cascade is a waveguide manifold with a short-circuit termination. Branches are connected to the main cascade through nonideal series junctions.

ducer loss, gain slope and group delay between commonport and channel output ports.

To apply the general method of Section II, the subnetworks, namely, channel filters, waveguide spacings, and junctions, should be represented by 2-port transmission matrices. Recently, a comprehensive set of formulas for reduction of multicavity filters to 2-port equivalents which also provides sensitivities w.r.t. variables of interest in the filter structure, has been presented [13]. The formulas evaluate short-circuit admittance parameters and their sensitivities w.r.t. all couplings as well as frequency for the unterminated filter model and take many nonideal effects such as losses, stray couplings, and dispersion into account. Evaluation of transmission matrices from short-circuit admittance matrices is straightforward. Here we describe the essential information in [13] which is appropriate to this paper.

The symmetrical impedance matrix for a narrow-band lumped model of an unterminated filter is given by

$$
\boldsymbol{Z}=j(s \mathbf{1}+\boldsymbol{M})+\boldsymbol{r} \mathbf{1}
$$

where 1 denotes an $n \times n$ identity matrix and $s$ is the normalized frequency variable given by

$$
s \triangleq \frac{\omega_{0}}{\Delta \omega}\left(\frac{\omega}{\omega_{0}}-\frac{\omega_{0}}{\omega}\right)
$$

$\omega_{0}$ and $\Delta \omega$ being the synchronously tuned cavity resonant frequency and the bandwidth parameter. We assume uniform dissipation for all cavities indicated by parameter $r$ where

$$
r=\frac{\omega_{0}}{\Delta \omega Q_{f}}
$$

$Q_{f}$ representing the unloaded $Q$-factor. In (27), $M$ is the coupling matrix whose $(i, j)$ element represents the normalized coupling between the $i$ th and $j$ th cavities and the diagonal entries $M_{n 1}$ represent the deviations from synchronous tuning. Dispersion effects on the filter are modeled by a frequency-dependent $\boldsymbol{M}$ matrix. It is easy to prove [13] that to calculate the short-circuit admittance parameters and their sensitivities w.r.t. all couplings and frequency, we
TABLE I

EXAMPLES OF TRANSMISSION MATRICES FOR SUBNETWORKS IN THE MULTIPLEXER OF FIG. 5

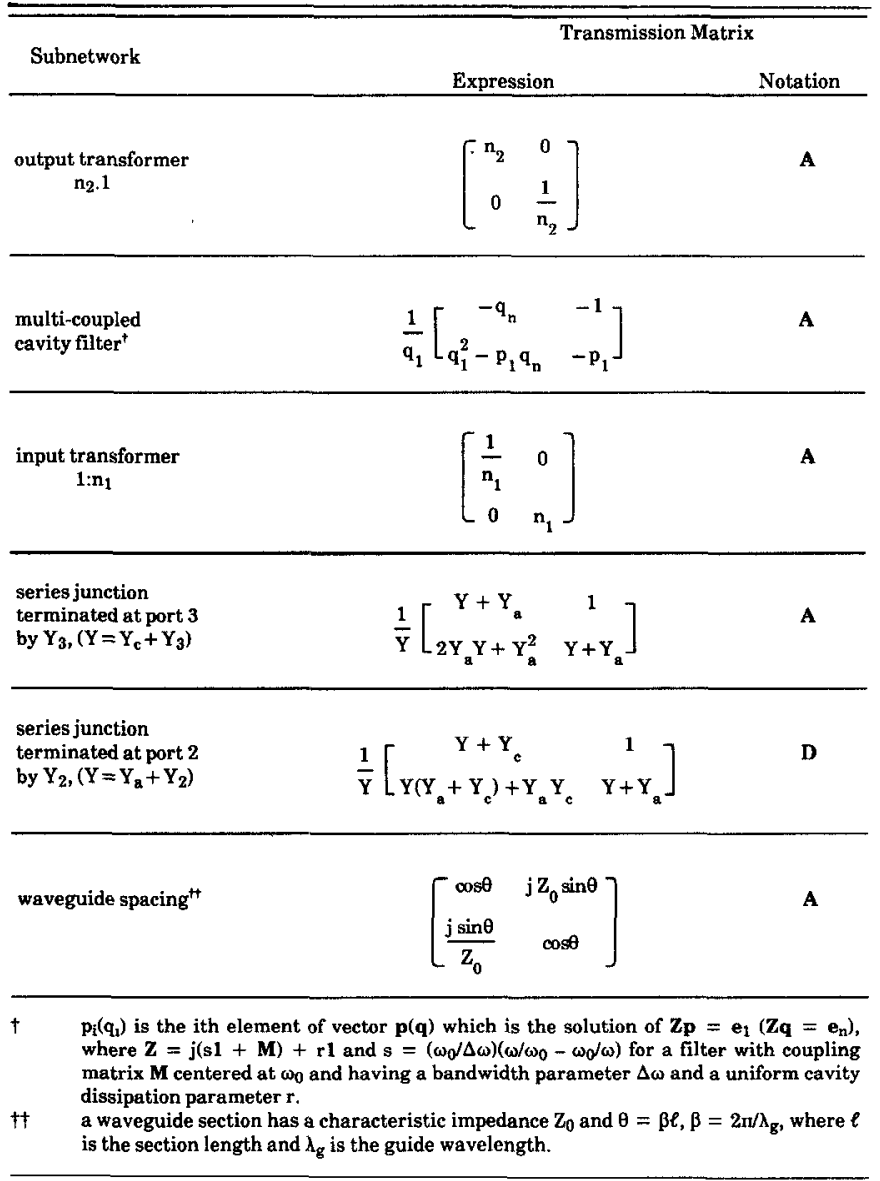

only need the solution vectors $\boldsymbol{p}$ and $\boldsymbol{q}$ of two systems of equations, namely,

$$
Z p=e_{1}
$$

and

$$
Z q=e_{n}
$$

where $e_{1}=\left[\begin{array}{ll}1 & 0 \cdots 0\end{array}\right]^{T}$ and $e_{n}=\left[\begin{array}{lll}0 & \cdots 0 & 1\end{array}\right]^{T}$.

In Table I, the transmission matrices for the individual components of the multiplexer structure shown in Fig. 5, have been listed. The series 3-port junctions are reduced to 2-port equivalents using the method described in Section II. Table II lists the sensitivities of transmission matrices in Table I w.r.t. relevant parameters and frequency.

In Table III, various frequency responses of interest in a multiplexer structure and their sensitivities w.r.t. design parameter $\phi$ have been summarized. It is clear that the evaluation of reflection coefficients at the common-port and channel output ports $\left(\rho^{0}\right.$ and $\left.\rho^{k}\right)$, channel output voltages $\left(V^{k}\right)$ and the first- and second-order sensitivities

$$
\left(\frac{\partial \rho^{0}}{\partial \phi}, \frac{\partial \rho^{k}}{\partial \phi}, \frac{\partial V^{k}}{\partial \phi}, \frac{\partial V^{k}}{\partial \omega} \text { and } \frac{\partial^{2} V^{k}}{\partial \phi \partial \omega}\right)
$$

as described in Section II, is sufficient to compute all responses and sensitivities tabulated. 
TABLE II

First-Order Sensitivities of the TRANSMission MATRICES IN TABLE I

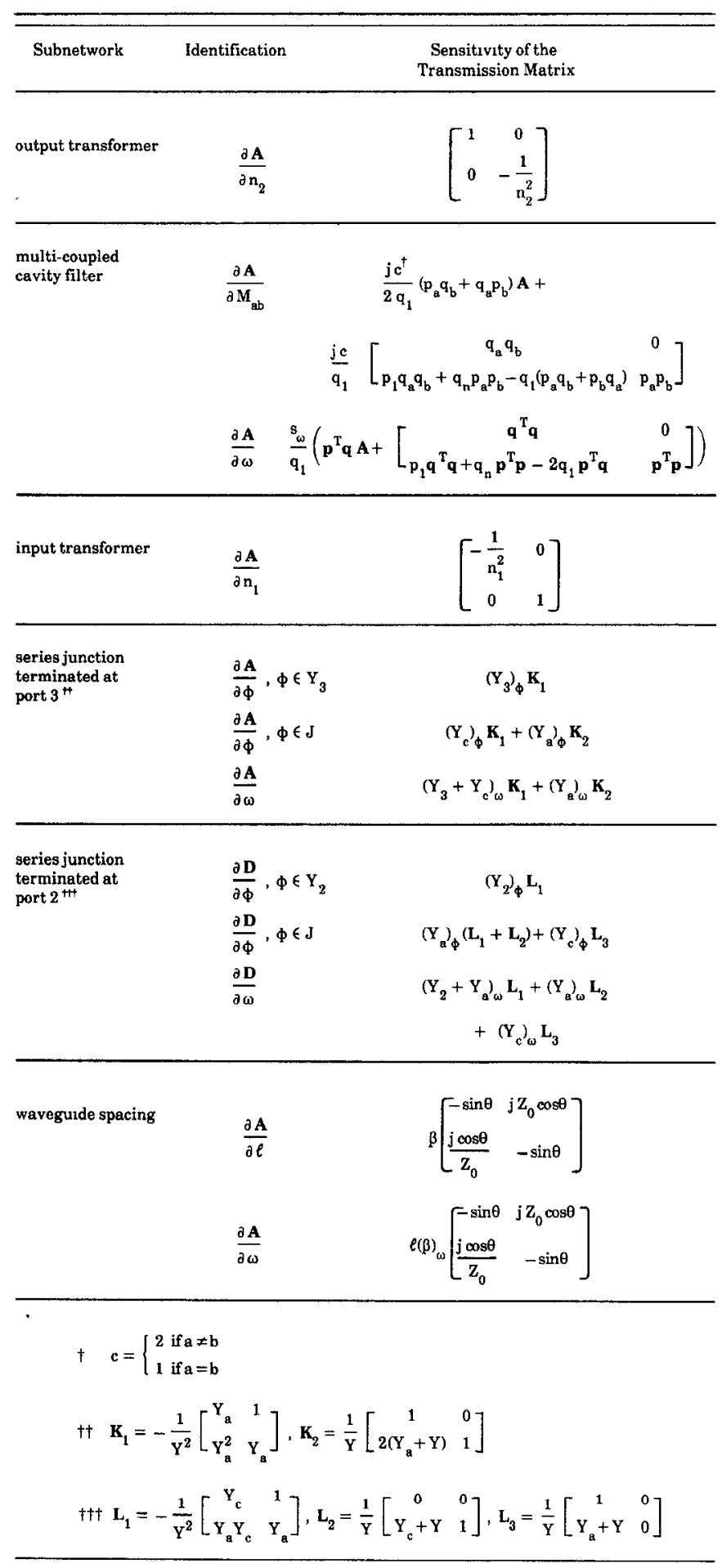

\section{Algorithm for Calculation of Thevenin Equivalents and Their Sensitivities}

The following algorithm can be used to obtain Thevenin equivalents at output ports and their sensitivities w.r.t. any variable. The algorithm assumes that the transmission matrices for all 2-port elements and the hybrid matrices for all junctions, as well as their sensitivities, are given. The
TABLE III

VARIOUS FREQUENCY RESPONSES AND THEIR SENSITIVITIES

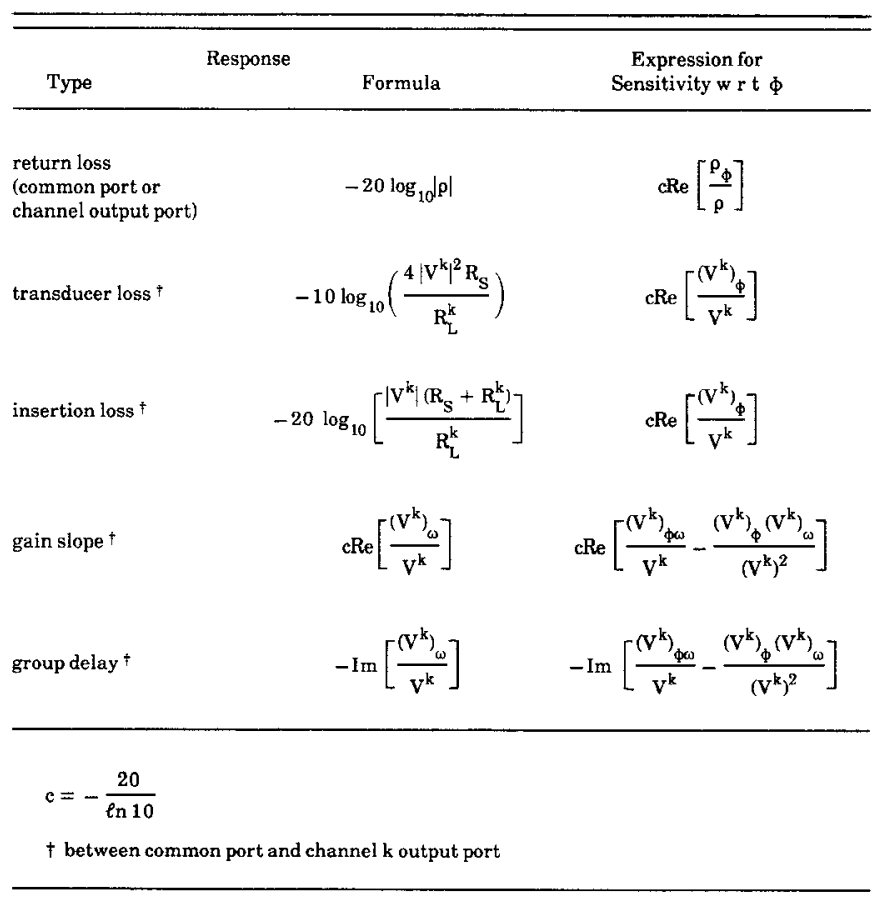

reverse analysis along the main cascade is initialized by $\boldsymbol{e}_{2}$ for a short-circuit termination or $\boldsymbol{e}_{1}$ for an open-circuit termination. Correspondingly, the resulting analysis is represented by $\boldsymbol{q}$ vectors (as in the algorithm) or $\boldsymbol{p}$ vectors as defined by (5).

Step 1 For $k=1,2, \cdots, N$, set $\sigma$ and $\tau$ to $\sigma(k)$ and $\tau(k)$, respectively, and execute Steps 1.1 to 1.7

Step 1.1 Calculate $Q_{l, \tau+1}$ by reverse analysis for $i=\tau+$ $1, \tau+2, \cdots, \sigma$. Calculate $Q_{\sigma,}$ by forward analysis for $j=\sigma, \sigma-1, \cdots, \tau+1$.

Comment Cascaded analysis is performed on the $k$ th branch. The reverse (forward) analysis starts from the branch output (input) port and is carried to the branch input (output) port.

Step $1.2 \quad \boldsymbol{p}_{\sigma \tau} \leftarrow \boldsymbol{Q}_{\sigma, \tau+1} \boldsymbol{A}_{\tau} \boldsymbol{e}_{1}$. Calculate $Y_{3}^{k}$ using (23).

Comment The equivalent admittance of the $k$ th branch, looking from the branch input port, is computed. This admittance is utilized in the 2-port representation of the $k$ th junction.

Step 1.3 Calculate $\partial \boldsymbol{p}_{\boldsymbol{\sigma} \tau} / \partial \phi$ using (6) and $\partial Y_{3}^{k} / \partial \phi$ from (22) for all the variable $\phi$ 's in the $k$ th branch.

Comment Sensitivities of the branch equivalent admittance w.r.t. all variables in the branch are calculated. In evaluating $\partial Y_{3}^{k} / \partial \phi$, we use a special case for (22) which corresponds to $Y_{3}^{k}$ given in (23).

Step 1.4 Calculate $\boldsymbol{A}_{2 k}$ using (3). Calculate $\partial \boldsymbol{A}_{2 k} / \partial \phi$ for all the variable $\phi$ 's in the $k$ th junction and the $k$ th branch.

Comment The 2-port representation of the $k$ th junction, when terminating its port 3 , is computed. The 
sensitivities of the resulting transmission matrix are readily obtained.

Step 1.5 Calculate $\boldsymbol{q}_{2 k, 1}$ by reverse analysis and $Y_{2}^{k}$ from (24).

Comment The equivalent admittance at port 2 of the $k$ th junction, looking towards the main cascade termination, is calculated after a reverse analysis from reference plane 1 to reference plane $2 k$.

Step 1.6 Calculate $\partial q_{2 k, 1} / \partial \phi$ using (6) and $\partial Y_{2}^{k} / \partial \phi$ using (22) for all variables $\phi$ in section $k^{\prime}, k^{\prime}<$ $k$ and in the $k$ th spacing.

Comment The sensitivities of the equivalent admittance $Y_{2}^{k}$, w.r.t. all variables geometrically located to the right of junction $k$, are computed. In evaluating $\partial Y_{2}^{k} / \partial \phi$, we use a special case for (22) which corresponds to $Y_{2}^{k}$, given in (24).

Step 1.7 Calculate $D_{2 k}$ using the method described in Section II. Calculate $\partial D_{2 k} / \partial \phi$ for all the variable $\phi$ 's in the $k$ th junction and spacing, as well as in all $k^{\prime}$ sections, $k^{\prime}<k$.

Comment The 2-port representation of junction $k$ when terminating its port 2 is computed. The sensitivities of the resulting transmission matrix w.r.t. all variables, included in or located to the right of the junction, are computed.

Step 2 Calculate $\boldsymbol{q}_{2 N+2,1}$ by extending the reverse analysis, already performed up to reference plane $2 N$ in Step 1.5, to reference plane $2 N+2$. Note that $\boldsymbol{A}_{2 N}$ has been evaluated in Step 1.4. Calculate $\partial \boldsymbol{q}_{2 N+2,1} / \partial \phi$ using $\partial \boldsymbol{q}_{2 N, 1} / \partial \phi(\phi$ belongs to the set of all variables to the right of section $N$ and the $N$ th spacing), which has been evaluated in Step 1.6, and $\partial A_{2 N} / \partial \phi(\phi$ belongs to the set of all variables in the $N$ th branch and $N$ th junction), which has been evaluated in Step 1.4.

Comment The reverse analysis from the main cascade termination is carried back to the source port. The corresponding sensitivities are also calculated. These results are used to calculate the common-port reflection coefficient and its sensitivities w.r.t. all variables in the entire network.

Step 3 For $k=N, N-1, \cdots, 1$, set $\sigma$ and $\tau$ to $\sigma(k)$ and $\tau(k)$, respectively, and execute Steps 3.1 to 3.3.

Step 3.1 Calculate $\boldsymbol{Q}_{2 N+2,2 k+1}$ by forward analysis.

Comment The forward analysis is carried along the main cascade from the source port to the input port of junction $k$.

Step $3.2 \quad \boldsymbol{Q}_{2 N+2, \tau+1} \leftarrow \boldsymbol{Q}_{2 N+2,2 k+1} \boldsymbol{D}_{2 k} \boldsymbol{Q}_{\boldsymbol{o}, \tau+1}$. Calculate $\partial \boldsymbol{Q}_{2 N+2, \tau+1} / \partial \phi$ using (6) for all the variable $\phi$ 's in the entire multiplexer.

Comment A cascaded analysis from the source port is carried through the $k$ th junction into the $k$ th branch. The sensitivities w.r.t. all variables are computed.
Step 3.3 Calculate $V_{S}^{\tau+1}$ and $Z_{S}^{\tau+1}$ using (8) and (9). Also, calculate $\partial V_{S}^{\tau+1} / \partial \phi$ and $\partial Z_{S}^{\tau+1} / \partial \phi$ using (10) and (11) for all variables $\phi$ in the entire multiplexer.

Comment Thevenin equivalents and their sensitivities are computed for the $k$ th branch output port.

\section{EXAMPLE}

A wide range of possible multiplexer optimization problems can be formulated and solved by appropriately defining specifications on various frequency responses of interest. The sensitivities are used in conjunction with the gradient-based minimax algorithm of Hald and Madsen [14] to ensure the fastest possible solutions.

As an example, we have used our simulation and sensitivity formulas to optimize a $12-\mathrm{channel}, 12-\mathrm{GHz}$ multiplexer without dummy channels. Waveguide spacings, input and output transformer ratios, cavity resonant frequencies, as well as intercavity couplings are used as optimization variables.

The details of the problem are as follows. There are twelve 6th-order multicavity filters mounted on the waveguide manifold. The transmission matrix deduced from the commonly used impedance matrix description of these filters has been formulated in Table I. An optimization on a singly terminated filter was performed to obtain the starting values for the nonzero couplings $M_{12}, M_{23}$, $M_{34}, M_{36}, M_{45}, M_{56}$, and the same values were assumed for all filters. The unloaded $Q$-factor is estimated at 12000 and dispersion effects consistent with the models in use at ComDev Ltd. [15] are included. In selecting the starting values of waveguide spacings, for each section the half-guide wavelength evaluated at the center frequency of the corresponding channel filter, as suggested in the literature [1], was used. The model for the nonideal junctions, i.e., the equivalent admittances $Y_{a}$ and $Y_{c}$ of Fig. 5, which have also been assumed in the transmission matrix description of junctions as appearing in Table $\mathrm{I}$, are consistent with the models suggested by Chen et al. [2] following the formulas by Marcuvitz [16]. Fig. 6 shows the common-port return loss and channel insertion-loss responses at the starting point for the optimization of the whole structure.

The specific optimization problem considered in this example was to satisfy a lower specification of $20 \mathrm{~dB}$ on the common-port return loss over the entire frequency band of interest for the multiplexer. From Table III, it is clear that the evaluation of common-port return loss and its sensitivities w.r.t. the generic optimization variable $\phi$ is straightforward once the common-port reflection coefficient $\rho^{0}$ and its sensitivities $\left(\rho^{0}\right)_{\phi}$ are known. We will describe the particular variables considered in this example later. Recalling (25) and (26) and the definition of $\boldsymbol{q}_{t}$ in (5), $\rho^{0}$ and $\left(\rho^{0}\right)_{\phi}$ are evaluated from $q_{2 N+2,1}$ and its sensitivities. Finally, by referring to the algorithm and specifically Step 2 in this case, $\boldsymbol{q}_{2 N+2,1}, \partial \boldsymbol{q}_{2 N+2,1} / \partial \phi$ are calculated.

The optimization was performed in several stages with the judicious addition of new variables at each stage to 


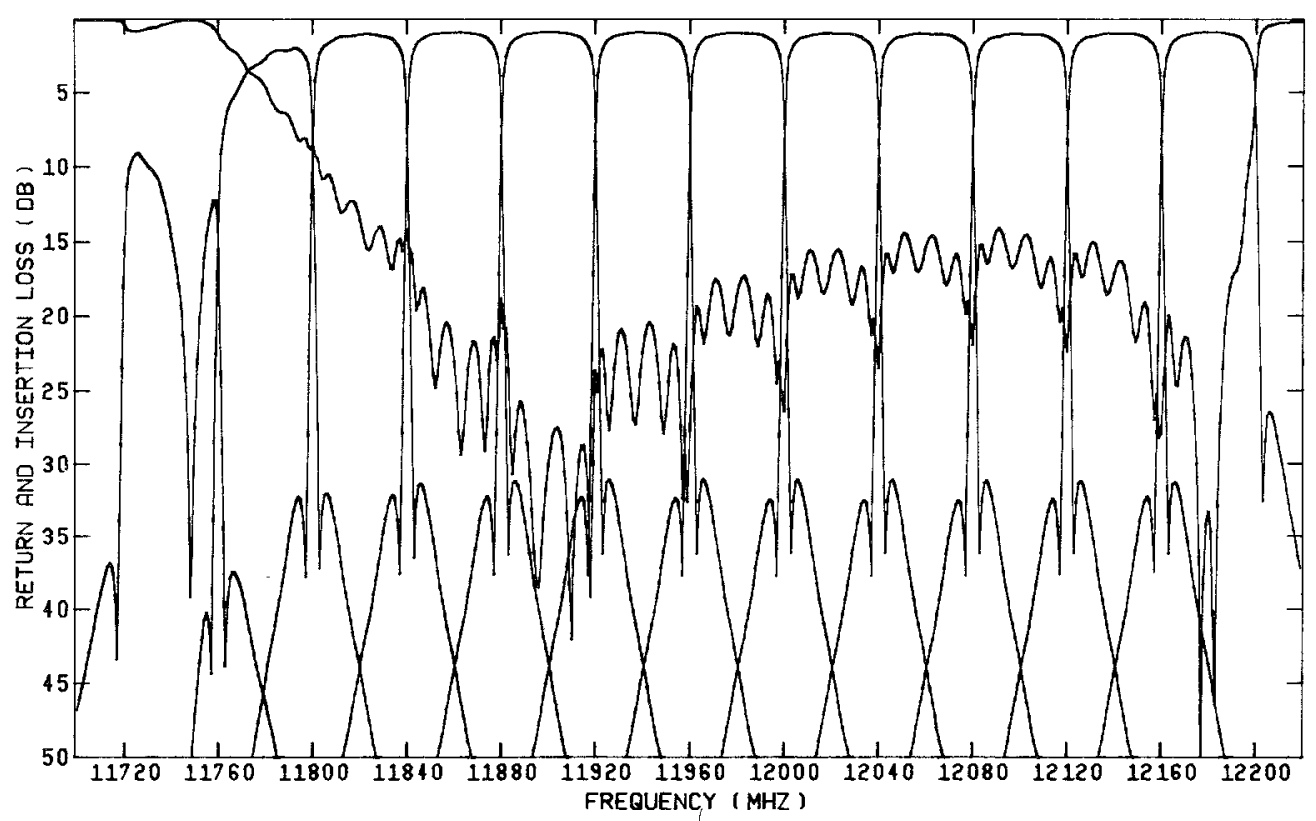

Fig. 6. Common-port return-loss and channel output port insertion-loss responses of the 12-channel multiplexer before optimization.

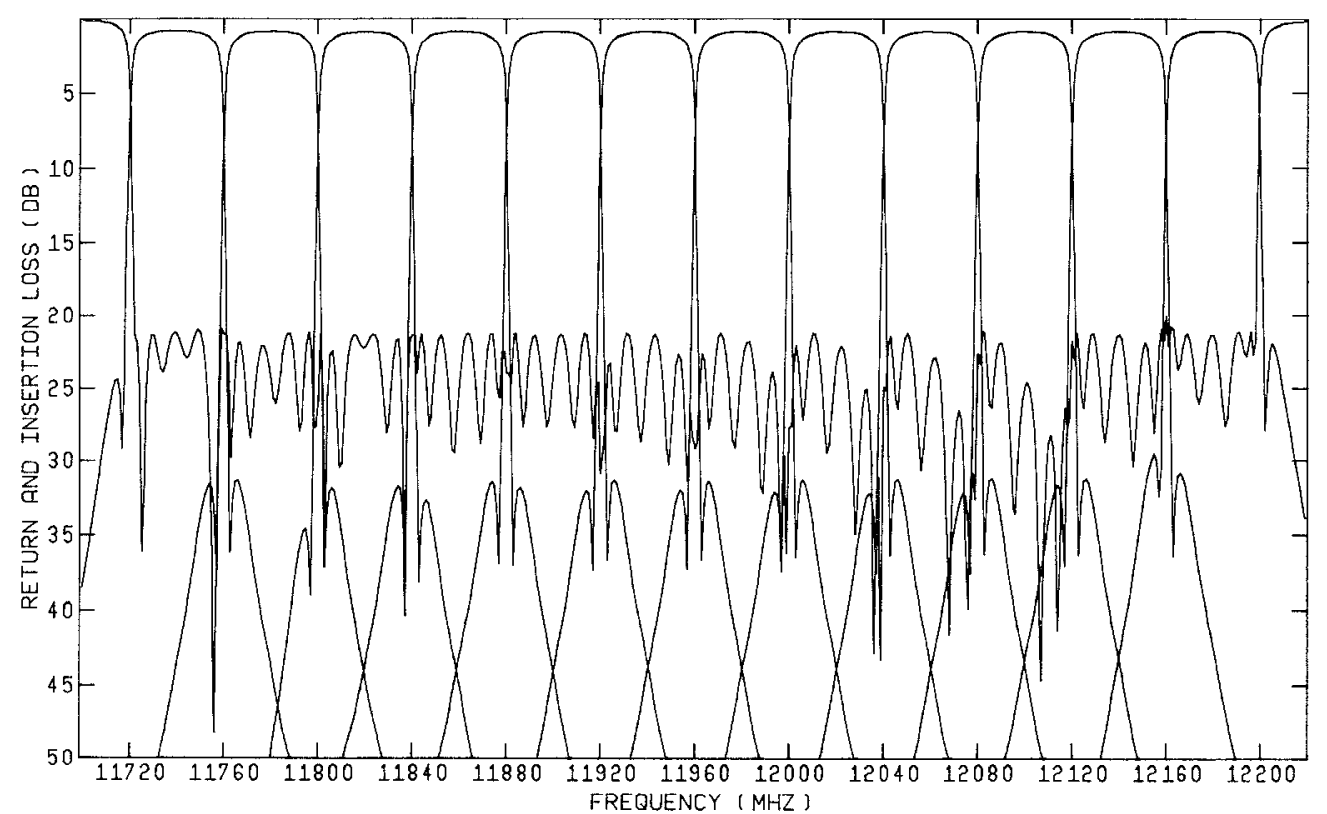

Fig. 7. Common-port return-loss and channel output port insertion-loss responses of the 12-channel multiplexer with optimized spacings, input-output transformer ratios, cavity resonances, and coupling parameters.

improve the overall response or the response over some specific portions of the total frequency band. In particular, the first stage was the optimization w.r.t. only waveguide spacings, i.e., 12 variables, and the last stage involved 60 variables, namely, 12 section lengths, 14 variables for each of channels 1 and 12 (all six possible intercavity couplings, six cavity resonant frequencies, input and output transformer ratios), and four variables for each of channels 2,8 , 9, 10, and 11 (input and output transformer ratios, resonant frequency of the first cavity, and coupling $M_{12}$ ). In selection of the frequency points, uniformly distributed points, $10 \mathrm{MHz}$ apart over the whole $500-\mathrm{MHz}$ band, are taken in the early stages. However, a simple interpolation technique effectively treating sample points $1 \mathrm{MHz}$ apart is introduced in the final stages of the optimization. The total CPU time on the Cyber 170/815 system was about ten minutes. The results of the final optimization are shown in Fig. 7. Equi-ripple return-loss response satisfying the requirements over the entire communication band has been achieved.

The results presented in this paper verify a highly efficient, state-of-the-art computer program package for simulation, sensitivity analysis, and optimization of multiplexers called MXSOS2. That package was developed by 
Optimizations Systems Associates [17] for ComDev Ltd. [15]. MXSOS2 was tested in close cooperation with members of ComDev Ltd., directly involved in multiplexer design and postproduction tuning [8].

\section{CONCLUSIONS}

We have presented a new approach to simulation and sensitivity analysis of multiplexing networks. By utilizing our formulas of Thevenin equivalents and their sensitivities w.r.t. network parameters as well as frequency, various frequency responses, and their sensitivities at arbitrarily chosen reference planes are evaluated. The method presented has been utilized in the optimal design of a state-ofthe-art 12-channel contiguous band multiplexer. Attractive and fast computer results obtained using a gradient-based optimization technique justify our treatment of sensitivity evaluation as an integral part of the analysis. All the sensitivity formulas presented in this paper can be verified independently. Actual implementation of our approach, however, requires only an understanding of the definitions of the responses, formulas for which are available in Table III. For more theoretically oriented researchers or engineers, our method of dealing with the sensitivities (Section II) is straightforward and should be applicable to almost any complex linear circuit structure in the frequency domain.

\section{ACKNOWLEDGMENT}

The authors are pleased to recognize the very strong support of this research program by M. V. O'Donovan (President) and Dr. C. M. Kudsia of ComDev Ltd, Cambridge, Ontario, Canada. The authors must also acknowledge useful technical discussions with $\mathrm{R}$. Tong and $\mathrm{L}$. Zaifman of the same company. The authors thank S. H. Chen of McMaster University for providing the unterminated filter simulation and sensitivity analysis formulas listed in Tables I and II. The authors also thank W. Kellermann of McMaster University for his cooperation in producing numerical results used in this paper.

\section{REFERENCES}

[1] A. E. Atia, "Computer-aided design of waveguide multiplexers," IEEE Trans. Microwave Theory Tech., vol. MTT-22, pp. 332-336, 1974.

[2] M. H. Chen, F. Assal, and C. Mahle, "A contiguous band multiplexer," COMSAT Tech. Rev., vol. 6, pp. 285-306, 1976.

[3] R. Tong, J. Dorey, P. Mabson, W. C. Tang, E. Klein-Lebbink, and C. M. Kudsia, "An $11 \mathrm{GHz}$ contiguous band output multiplexing network for Intelsat VI spacecraft," in 1982 IEEE Int. Microwave Symp. Dig. (Dallas, TX), 1982, pp. 405-407.

[4] M. H. Chen, "A 12-channel contiguous band multiplexer at $\mathrm{Ku}$ band," in 1983 IEEE Int. Microwave Symp. Dig. (Boston, MA), 1983, pp. 77-79.

[5] R. G. Egri, A. E. Williams, and A. E. Atia, "A contiguous-band multiplexer design," in 1983 IEEE. Int. Microwave Symp. Dig. (Boston, MA), 1983, pp. 86-88.

[6] S. C. Holme, "A $12 \mathrm{GHz} 12$ channel multiplexer for satellite applications," in 1984 IEEE Int. Microwave Symp. Dig. (San Francisco, CA), 1984, pp. 295-296.

[7] M. H. Chen, "Current state-of-the-art technology on contiguousband multiplexer," in Proc. IEEE Int. Symp. Circuits and Systems (Kyoto, Japan), 1985, pp. 1583-1586.

[8] J. W. Bandler, S. H. Chen, S. Daijavad, and W. Kellermann, "Optimal design of multicavity filters and contiguous-band multi- plexers," in Proc. 14th European Microwave Conf. (Liège, Belgium), 1984, pp. $863-868$.

[9] J. W. Bandler, W. Kellermann, and K. Madsen, "A superlinearly convergent minimax algorithm for microwave circuit design," IEEE Trans. Microwave Theory Tech.; vol. MTT-33, pp. 1519-1530, Dec. 1985.

[10] J. W. Bandler, S. Daijavad, and Q. J. Zhang, "Computer aided design of branched cascaded networks," in Proc. IEEE Int. Symp. Circuits and Systems (Kyoto, Japan), 1985, pp. 1579-1582.

[11] J. W. Bandler, M. R. M. Rizk, and H. L. Abdel-Malek, "New results in network simulation, sensitivity, and tolerance analysis for cascaded structures," IEEE Trans. Microwave Theory Tech., vol. MTT-26, pp. 963-972, 1978.

[12] J. W. Bandler and M. R. M. Rizk, "Tolerance analysis of cascaded structures," IEEE Trans. Microwave Theory Tech., vol. MTT-28, pp. $935-938,1980$

[13] J. W. Bandler, S. H. Chen, and S. Daijavad, "Exact sensitivity analysis for optimization of multi-coupled cavity filters," Int. J. Circuit Theory Appl., to be published.

[14] J. Hald and K. Madsen, "Combined LP and quasi-Newton methods for minimax optimization," Mathematical Programming, vol. 20, pp. 49-62, 1981.

[15] ComDev Ltd., 155 Sheldon Drive, Cambridge, Ontario, Canada N1R 7H6, private communications, 1983-1984.

[16] N. Marcuvitz, Waveguide Handbook (MIT Rad. Lab. Ser., vol. 10). New York: McGraw-Hill, 1951

[17] Optimization Systems Associates, 163 Watson's Lane, Dundas, Ontario, Canada L9H 6L1, 1983--1984.

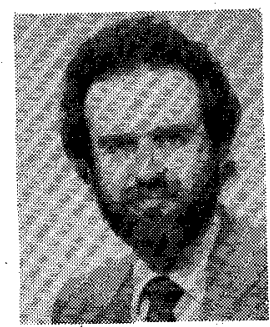

John W. Bandler (''66-M'66-SM'74-F'78) was born in Jerusalem, Palestine, on November 9, 1941. He studied at the Imperial College of Science and Technology, London, England, from 1960-1966. He received the B.Sc. (Eng.), Ph.D., and D.Sc. (Eng.) degrees from the University of London, London, England, in 1963, 1967, and 1976, respectively

$\mathrm{He}$ joined Mullard Research Laboratories, Redhill; Surrey, England, in 1966. From 1967 to 1969, he was a Postdoctorate Fellow and Sessional Lecturer at the University of Manitoba, Winnipeg, Canada. He joined McMaster University, Hamilton, Canada, in 1969, where he is currently a Professor of Electrical and Computer Engineering. He has served as Chairman of the Department of Electrical Engineering and Dean of the Faculty of Engineering. He currently directs research, which has received substantial support by the Natural Sciences and Engineering Research Council of Canada under its Operating and Strategic Grants Awards, in the Simulation Optimization Systems Research Laboratory.

Dr. Bandler is also currently President of Optimization Systems Associates. He has provided consulting services and software to numerous organizations in the electronic, microwave, and electrical power industry, specializing in advanced applications of simulation, sensitivity analysis, and mathematical optimization techniques.

He is a contributor to Modern Filter Theory and Design, Wiley-Interscience, 1973. He has over 200 publications, four of which appear in Computer-Aided Filier Design, IEEE Press, 1973, one in Microwave Integrated Circuits, Artech House, 1975, and one in Low-Noise Microwave Transistors and Amplifiers, IEEE Press, 1981. Dr: Bandler was an Associate Editor of the IEEE TRANSACTIONS ON Microwave THEORY AND TECHNIOUES (1969-1974). He was Guest Editor of the Special Issue of the IEEE TRansactions on Microwave Theory and TeChNiques on Computer-Oriented Microwave Practices (March 1974).

Dr. Bandler is a Fellow of the Institution of Electrical Engineers (Great Britain) and a member of the Association of Professional Engineers of the Province of Ontario (Canada). 


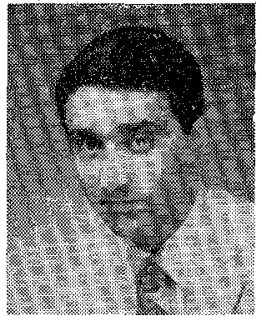

Shahrokh Daijavad (S'82) was born in Isfahan, Iran, on July 7, 1960. He received the B.Eng. degree in electrical engineering (summa cum laude) from McMaster University, Hamilton, Ontario, Canada, in 1983, and was awarded the gold medal for academic achievement by the Association of Professional Engineers of Ontario.

$\mathrm{He}$ is now completing the Ph.D. degree in electrical engineering at McMaster University, where he has been working since 1983 in the Simulation Optimization Systems Research

Laboratory.

During his doctoral studies, he has held an Ontario Graduate Scholarship and a Teaching Assistantship. He was awarded a Natural Sciences and Engineering Research Council of Canada Scholarship in 1985.

His research interests are in computer-aided circuit design with emphasis on applications of optimization theory in modeling, fault location, and tuning.

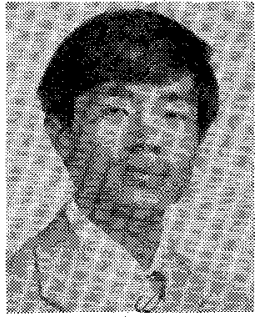

Qi-jun Zhang (S'84) was born in Xiangyan, Shanxi, China, on October 8, 1959. He received the B.Eng. degree from East China Engineering Institute, Nanjing, China, in 1982.

From February 1982 to August 1983, he was with the Research Institute of Systems Engineering, Tianjin University, Tianjin, China, as a research assistant. He was awarded a Chinese Government Scholarship and joined the Department of Electrical and Computer Engineering, McMaster University, Hamilton, Canada, in September 1983. He is currently carrying out research in the Simulation Optimization Systems Research Laboratory, McMaster University, where he is also a teaching assistant and graduate student working towards the $\mathrm{Ph}$.D. degree. He has been awarded an Ontario Graduate Scholarship for the academic year $1985 / 1986$.

His interests are in design optimization, sensitivity analysis, identification, and tuning. 